\title{
DUALITY OF THE CONES OF DIVISORS AND CURVES
}

\author{
Sung RAK ChOI
}

\begin{abstract}
S. Payne asked whether for a variety $X$ of dimension $d$, the closed cone spanned by the divisors ample in dimension $k(1 \leq k \leq d)$ and the closed cone spanned by the classes of curves on some $\mathbb{Q}$-factorial small modifications of $X$ movable in codimension $d-k$ are dual to each other. We prove that this is true for Fano type varieties and Mori dream spaces.
\end{abstract}

\section{Introduction}

By a variety throughout the paper, we mean a normal projective algebraic variety defined over an algebraically closed field of characteristic 0 (e.g., the complex numbers $\mathbb{C}$ ). For a $\mathbb{Q}$-divisor $D$ on a variety $X$, the stable base locus $\mathbf{B}(D)$ is defined as the set theoretic intersection of all the base loci of $|m D|$ for positive integers $m$ such that $m D$ are integral. For an integer $k$ such that $1 \leq k \leq d=\operatorname{dim} X$, we let $\overline{\operatorname{Amp}}_{k}(X)$ be the closed cone in $\mathrm{N}^{1}(X)$ spanned by the $\mathbb{Q}$-divisors $D$ such that the stable base loci $\mathbf{B}(D)$ do not contain any $k$-dimensional subvarieties of $X$ (Definition 4.3, Theorem 4.1). Let $f: X \rightarrow X^{\prime}$ be an isomorphism in codimension 1 between $\mathbb{Q}$-factorial varieties $X$ and $X^{\prime}$. It is well known that $\mathrm{N}^{1}(X) \cong \mathrm{N}^{1}\left(X^{\prime}\right)$ and consequently $\mathrm{N}_{1}(X) \cong \mathrm{N}_{1}\left(X^{\prime}\right)$ by dualizing. Thus a curve $C^{\prime}$ on $X^{\prime}$ defines a class $\alpha$ in $\mathrm{N}_{1}(X)$ even though the class $\alpha$ may not represent a well-defined curve on $X$. Assume further that there exists a subvariety $V^{\prime} \subseteq X^{\prime}$ such that $f^{-1}$ is isomorphic at the generic point of $V^{\prime}$. If $V^{\prime}$ is $k$-codimensional, then a curve $C^{\prime}$ on $X^{\prime}$ belonging to a family of curves covering $V^{\prime}$ is called a $b-m o v^{k}$-curve of $X$ (Definition 4.1). As explained above, a b-mov ${ }^{k}$-curve of $X$ naturally defines a class in $\mathrm{N}_{1}(X)$. Define $\overline{\mathrm{bNM}}^{k}(X)$ be the closed convex cone in $\mathrm{N}_{1}(X)$ spanned by the classes of b-mov ${ }^{k}$-curves of $X$.

S. Payne asks if the following is true.

Problem 1.1. [17, Problem] Let $X$ be a $\mathbb{Q}$-factorial projective variety of dimension $d$. Then for integers $k$ such that $1 \leq k \leq d$, the following duality holds:

$$
\overline{\operatorname{Amp}}_{k}(X)^{\vee}=\overline{\mathrm{bNM}}^{d-k}(X) \text {. }
$$

(Payne in [17] uses the reverse notation. He uses the codimension for the cone of divisors and dimension for the cone of curves.)

The duality is well known for $k=1$ due to Kleiman-Seshadri and for $k=d$ due to Boucksom-Demaillly-Paun-Peternell (Theorem 4.2). It is proven in [17] that the above equality holds for all $k$ if $X$ is a complete $\mathbb{Q}$-factorial toric variety. As pointed out in [17] with an example, it is important to note that considering the curves only on $X$ is not enough in the duality of Problem 1.1.

Received by the editors June 15, 2011. 
A Fano type $(F T)$ variety $X$ is a variety for which there exists a $\mathbb{Q}$-boundary divisor $B$ such that $(X, B)$ is a log Fano pair (Definition 4.4$)$. We prove the following:

Theorem 1.1. (=Corollary 4.2) Let $X$ be a $\mathbb{Q}$-factorial FT variety of dimension $d$. Then the duality of Problem 1.1 holds for all integers $k$ such $1 \leq k \leq d$.

We will first prove that the duality of Problem 1.1 holds in the $(K+B)$-negative part for a klt pair $(X, B)$ using the result in the log minimal model program (LMMP for short) (Theorem 4.3). The case $k=d-1$ is proven in [6]. For the general case where $k<d-1$, we need to keep track of the irreducible components of codimension $>1$ of the base locus of $K+B$ while we run the LMMP (Theorem 3.2) and we prove the duality by induction. On an FT variety $X$, there exists a boundary divisor $B^{\prime}$ such that $\left(X, B^{\prime}\right)$ is log Fano (Definition 4.4). Thus, the cones $\overline{\operatorname{Amp}}_{k}(X)$ entirely lie in the $(K+B)$-negative part and we obtain the duality of Problem 1.1. We can also prove that the duality also holds for a Mori dream space.

Corollary 1.1. (=Corollary 4.3) Let $X$ be a Mori dream space of dimension d. Then the duality of Problem 1.1 holds for all integers $k$ such $1 \leq k \leq d$.

In Section 2, we gather some results on the non-ample locus $\mathbf{B}_{+}(D)$ and the nonnef locus $\mathbf{B}_{-}(D)$. In Section 3, we study how the modifications of LMMP affect the components of $\mathbf{B}_{+}(D)$ and $\mathbf{B}_{-}(D)$. In Section 4, we prove Theorem 1.1.

\section{Preliminaries}

Let $X$ be a $\mathbb{Q}$-factorial normal projective variety. For a $\mathbb{Q}$-divisor $D$ on $X$, the stable base locus is defined as $\mathbf{B}(D):=\cap \mathrm{Bs}|m D|$ where the intersection is taken over all positive integers $m$ such that $m D$ are integral. Let $D$ be an $\mathbb{R}$-divisor on $X$. The non-ample locus (or augmented base locus) $\mathbf{B}_{+}(D)$ of $D$ is defined as

$$
\mathbf{B}_{+}(D):=\bigcap \mathbf{B}(D-A)
$$

where the intersection is taken over all ample divisors $A$ on $X$ such that $D-A$ are $\mathbb{Q}$-divisors. The non-nef locus (or diminished base locus) $\mathbf{B}_{-}(D)$ of $D$ is defined as

$$
\mathbf{B}_{-}(D):=\bigcup \mathbf{B}(D+A),
$$

where the union is taken over all ample divisors $A$ on $X$ such that $D+A$ are $\mathbb{Q}$-divisors. For $\square=+$ or - , we define

$\operatorname{dim} \mathbf{B}_{\square}(D):=\max \left\{\operatorname{dim} V \mid V\right.$ is a subvariety of $X$ such that $\left.V \subseteq \mathbf{B}_{\square}(D)\right\}$.

By [7, Proposition 1.1], the loci $\mathbf{B}_{+}(D), \mathbf{B}_{-}(D)$ do not have any isolated points. Thus $\operatorname{dim} \mathbf{B}_{\square}(D)=0$ implies $\mathbf{B}_{\square}(D)=\emptyset$.

Definition 2.1. Let $X$ be a $d$-dimensional variety and $k$ be an integer such that $1 \leq k \leq d$. A divisor $D$ such that $\operatorname{dim} \mathbf{B}_{+}(D)<k$ is said to be ample in dimension $k$ and a divisor $D$ such that $\operatorname{dim} \mathbf{B}_{-}(D)<k$ is said to be nef in dimension $k$.

Note that a divisor ample (resp. nef) in dimension 1 is ample (resp. nef) and a divisor ample (resp. nef) in dimension $d$ is big (resp. pseudo-effective) in the usual sense. We call a divisor nef in dimension $d-1$ a mobile divisor.

Definition 2.2. When $\mathbf{B}_{-}(D) \neq X\left(\right.$ or $\left.\mathbf{B}_{+}(D) \neq X\right)$, its irreducible component $V$ is said to be divisorial if $\operatorname{dim} V=\operatorname{dim} X-1$ and small if $\operatorname{dim} V<\operatorname{dim} X-1$. 
One should distinguish the subvarieties $V$ which belong properly to a component of the base locus $\mathbf{B}_{\square}(D)(\square=+$ or - ) from the ones that are irreducible components of $\mathbf{B}_{\square}(D)$ (cf. [7, Example 5.10]). By an irreducible component $V$ of $\mathbf{B}_{\square}(D)$, we mean a maximal subvariety $V \subseteq \mathbf{B}_{\square}(D)$ in the sense that for a subvariety $V^{\prime}$ of $X, V \subsetneq V^{\prime}$ implies $V^{\prime} \nsubseteq \mathbf{B}_{\square}(D)$.

Remark 2.1. For a sufficiently small ample divisor $A$, we have $\mathbf{B}_{+}(D)=\mathbf{B}(D-A)$ [8, Proposition 1.5]. It is also easy to see that for any subvariety $V \subseteq \mathbf{B}_{-}(D)$, there exists a sufficiently small ample divisor $A$ such that $V \subseteq \mathbf{B}(D+A)\left(\right.$ or $\left.\mathbf{B}_{-}(D+A)\right)$.

The non-ample locus $\mathbf{B}_{+}(D)$ is Zariski closed, but it is not expected in general that the non-nef locus $\mathbf{B}_{-}(D)$ is also Zariski closed. Nonetheless, the base locus $\mathbf{B}_{-}(D)$ is a union of at most countable many Zariski closed subsets and contains only finitely divisorial components $[3,16]$.

Remark 2.2. We will often use the following inclusions without reference: for a sufficiently small ample divisor $A$,

$$
\mathbf{B}_{+}(D+A) \subseteq \mathbf{B}_{-}(D), \quad \mathbf{B}(D+A) \subseteq \mathbf{B}_{-}(D) \text { and } \quad \mathbf{B}_{+}(D+A) \subseteq \mathbf{B}(D) .
$$

(The proofs are easy and left to the readers.) Therefore, we can also define the non-nef locus as $\mathbf{B}_{-}(D):=\cup \mathbf{B}_{+}(D+A)$ where the union is taken over all ample divisors $A$.

The results below are useful when we study the base loci $\mathbf{B}_{+}$and $\mathbf{B}_{-}$. Nakamaye gives another characterization of the non-ample locus $\mathbf{B}_{+}(D)$ when $D$ is nef. We define the null locus $\operatorname{Null}(D)$ of a nef and big divisor $D$ as $\operatorname{Null}(D):=\bigcup_{V}\{V \subseteq$ $X \mid D^{k} \cdot V=0$, where $\left.\operatorname{dim} V=k>0\right\}$.

Theorem 2.1 (Nakamaye's theorem). [14, Theorem 10.3.5], [15] Let D be a nef divisor on $X$. Then

$$
\mathbf{B}_{+}(D)=\operatorname{Null}(D) .
$$

This implies $D^{\operatorname{dim} V} \cdot V=0$ for each irreducible component $V$ of $\mathbf{B}_{+}(D)$. The following describes how $\mathbf{B}_{+}, \mathbf{B}_{-}$vary under pull backs of birational morphisms.

Lemma 2.1. Let $f: X \rightarrow Y$ be a birational morphism of normal projective varieties. Then for a big divisor $D$ on $Y$ and any effective $f$-exceptional divisor $F$ on $X$, the following holds;

$$
\mathbf{B}_{+}\left(f^{*}(D)+F\right)=f^{-1}\left(\mathbf{B}_{+}(D)\right) \cup \operatorname{Exc}(f) .
$$

Proof. See [4, Proposition 1.5].

The non-nef locus $\mathbf{B}_{-}(D)$ can be described in terms of divisorial valuations.

Definition 2.3. Let $\sigma$ be a divisorial valuation of $X$ and $D$ be a pseudo-effective divisor on $X$. We define the asymptotic numerical divisorial valuation $\sigma_{\text {num }}(D)$ of $D$ at $\sigma$ as

$$
\sigma_{\text {num }}(D):=\inf \left\{\sigma\left(D^{\prime}\right) \mid D \equiv D^{\prime} \geq 0\right\} \quad \text { if } D \text { is big }
$$

and $\sigma_{\text {num }}(D):=\lim _{\varepsilon \rightarrow 0} \sigma_{\text {num }}(D+\varepsilon A)$ for some fixed ample divisor $A$ if $D$ is only pseudo-effective on $X$.

The definition is independent of the choice of $A$. 
Lemma 2.2. Let $f: W \rightarrow X$ be a birational morphism of normal projective varieties and $D$ be a divisor on $X$. Then for any divisorial valuation $\sigma$ of $X$ and for any effective $f$-exceptional divisor $F$ on $W$, we have

$$
\sigma_{n u m}\left(f^{*} D+F\right)=\sigma_{n u m}(D)+\sigma(F) .
$$

In particular, $\sigma_{\text {num }}\left(f^{*} D\right)=\sigma_{\text {num }}(D)$ ([4, Lemma 1.6]).

Proof. Let $E$ be an effective divisor on $W$ such that $E \equiv f^{*} D+F$. Then by the Negativity Lemma ([12, Lemma 3.39]), $E$ has the form $E=E^{\prime}+F$ where $E^{\prime} \equiv f^{*} D$. Thus $\sigma_{\text {num }}\left(f^{*} D+F\right)=\inf \left\{\sigma\left(E^{\prime}\right)+\sigma(F) \mid E^{\prime} \equiv f^{*} D\right\}$.

Proposition 2.1. Let $(X, B)$ be a projective klt pair. Then the equality holds:

$$
\mathbf{B}_{-}(K+B)=\bigcup_{\sigma}\left\{\operatorname{Cent}_{X} \sigma \mid \sigma_{n u m}(K+B)>0\right\}
$$

where the union is taken over all divisorial valuations $\sigma$ of $X$.

Proof. See [4, Proposition 1.10].

This implies that if $V$ is an irreducible component of $\mathbf{B}_{-}(K+B)$, then there exists a divisorial valuation $\sigma$ such that $\operatorname{Cent}_{X} \sigma=V$. By [8, Proposition 2.8], the equality in Proposition 2.1 holds for any divisor $D$ instead of $K+B$ when $X$ is smooth. We will need the following.

Corollary 2.1. Let $(X, B)$ be a projective klt pair such that $K+B$ is big. Then for some sufficiently small ample divisor $H$ on $X$,

$$
\mathbf{B}_{-}(K+B-H)=\bigcup\left\{\operatorname{Cent}_{X} \sigma \mid \sigma_{n u m}(K+B-H)>0\right\},
$$

where the union is taken over all divisorial valuations $\sigma$ of $X$.

Proof. Since $K+B$ is big, there exist an ample divisor $A$ and an effective divisor $E$ such that $K+B \equiv A+E$. Thus, we have

$$
\begin{aligned}
K+B-H & =K+B-\varepsilon A+\varepsilon A-H \\
& \equiv A+E-\varepsilon A+H^{\prime} \quad\left(\text { where } H^{\prime}=\varepsilon A-H\right) \\
& \equiv(1-\varepsilon)(A+E)+\varepsilon E+H^{\prime} \\
& \equiv(1-\varepsilon)\left(K+B+\frac{\varepsilon}{1-\varepsilon} E+\frac{1}{1-\varepsilon} H^{\prime}\right) .
\end{aligned}
$$

For a fixed sufficiently small $\varepsilon>0,\left(X, B+\frac{\varepsilon}{1-\varepsilon} E\right)$ is klt. By choosing $H$ sufficiently small, we may assume that $H^{\prime}=\varepsilon A-H$ is ample. Therefore, we may assume that the pair $\left(X, B+\frac{\varepsilon}{1-\varepsilon} E+\frac{1}{1-\varepsilon} H^{\prime}\right)$ is also klt. Since the locus $\mathbf{B}_{-}(D)$ and the positivity of $\sigma_{\text {num }}(D)$ are independent of rescaling $D$ by a positive number, Proposition 2.1 implies the desired equality.

See $[8,7,14,20]$, etc for more details on the non-ample and non-nef loci. 


\section{Base loci under birational maps}

We will follow the standard notions of singularities of the pairs $(X, B)$ in the LMMP $[2,10,12]$. For an exceptional prime divisor $E$ over $X$, the $\log$ discrepancy of $(X, B)$ at $E$ is denoted by $a(E, X, B)$. We recall the basic models treated in the LMMP.

Definition 3.1. Let $f: X \rightarrow Y$ a birational map of projective varieties and $(X, B)$ a klt pair. Then the map $f$ (or simply the variety $Y$ ) is called

(1) a log minimal model of $(X, B)$ if for $f_{*} B=B_{Y},\left(Y, B_{Y}\right)$ is klt, $K_{Y}+B_{Y}$ is nef and $1-$ mult $_{E} B<a\left(E, Y, B_{Y}\right)$ for any divisor $E$ exceptional over $Y$, and

(2) a log canonical model of $(X, B)$ if for $f_{*} B=B_{Y},\left(Y, B_{Y}\right)$ is lc, $K_{Y}+B_{Y}$ is ample and $1-$ mult $_{E} B \leq a\left(E, Y, B_{Y}\right)$ for any divisor $E$ exceptional over $Y$. Such model is unique and we denote the $\log$ canonical model of $(X, B)$ by $X_{\text {can }}$.

It is expected that a $\log$ minimal model $f: X \rightarrow Y$ of a pair $(X, B)$ can be decomposed into finitely many divisorial contractions and log flips.

Definition 3.2 (Log flip). Let $(X, B)$ be a projective klt pair and $\varphi: X \rightarrow Z$ be a birational contraction such that i) $\operatorname{codim} \operatorname{Exc}(\varphi) \geq 2$, ii $)-(K+B)$ is $\varphi$-ample, and iii) the relative Picard number $\rho(X / Z):=\rho(X)-\rho(Z)=1$. A modification $\chi: X \rightarrow X^{+}$ such that the pair $\left(X^{+}, B_{X^{+}}\right)$is klt where $B_{X^{+}}:=\chi_{*} B$ is called a $\log$ flip of $\varphi$ if there exists a contraction $\varphi^{+}: X^{+} \rightarrow Z$ satisfying the following properties:

(i') $\operatorname{codim} \operatorname{Exc}\left(\varphi^{+}\right) \geq 2$ and $\chi: X \backslash \operatorname{Exc}(\varphi) \cong X^{+} \backslash \operatorname{Exc}\left(\varphi^{+}\right)$is an isomorphism,

(ii') $K_{X^{+}}+B_{X^{+}}$is $\varphi^{+}$-ample, and

(iii') $\rho\left(X^{+} / Z\right)=1$ :

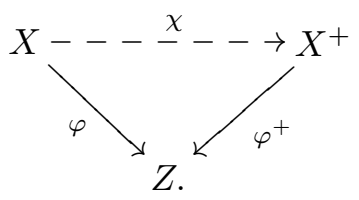

It is easy to see that the loci $\mathbf{B}_{+}(D)$ and $\mathbf{B}_{-}(D)$ are empty if and only if $D$ is ample and nef, respectively. In particular, for a projective klt pair $(X, B)$ such that $K+B$ is big, if $f: X \rightarrow Y$ is a $\log$ minimal model of $(X, B)$, then $\mathbf{B}_{-}\left(K_{Y}+B_{Y}\right)=\emptyset$ and if $X_{\text {can }}$ is the $\log$ canonical model of $(X, B)$, then $\mathbf{B}_{+}\left(K_{X_{\text {can }}}+B_{X_{\text {can }}}\right)=\emptyset$. Thus, the LMMP can be considered as a birational map which modifies the locus $\mathbf{B}_{-}(K+B)$ into nef on $Y$ and the $\log$ canonical (Iitaka) contraction $g: Y \rightarrow X_{\text {can }}$ as the morphism contracting the locus $\mathbf{B}_{+}\left(K_{Y}+B_{Y}\right)$ so that $K_{X_{\text {can }}}+B_{X_{\text {can }}}=g_{*}\left(K_{Y}+B_{Y}\right)$ becomes ample. In some sense, the LMMP and the log canonical (Iitaka) contraction are exactly the maps that perform such tasks. This is more or less known to the experts and can be found in the literature.

The following is now a basic result in the LMMP.

Theorem 3.1. [2, 11] Let $(X, B)$ be a projective klt pair such that $K+B$ is big. Then there exists a log minimal model $\varphi: X \rightarrow X_{\min }$ of $(X, B)$ such that

(1) $\varphi$ is surjective in codimension 1 , and

(2) a prime divisor $E$ of $X$ is contracted by $\varphi$ if and only if $E$ is a divisorial component of $\mathbf{B}_{-}(K+B)$. 
If furthermore $K+B$ is big, then there exists a log canonical model $X_{\text {can }}$ of $(X, B)$.

The number of divisorial components of $\mathbf{B}_{-}(D)$ for a pseudoeffective divisor $D$ is bounded by the Picard number $\rho(X)$ [3, Theorem 3.12]. In particular, it is finite and the number of divisorial components of $\mathbf{B}_{-}(K+B)$ is at most the number of divisorial contractions to be performed while we run the LMMP on $(X, B)$. In Theorem 3.3, we will prove the analogous statement for the divisorial components of $\mathbf{B}_{+}(K+B)$ over the $\log$ canonical model using the next results just for the completeness.

Definition 3.3. A birational map $f: X \rightarrow X^{\prime}$, which is an isomorphism in codimension 1 is said to be small. Assume that a small birational map $f$ is an isomorphism at the generic point of some positive dimensional subvariety $V \subseteq X$. Then there exists a subvariety $V^{\prime}$ of $X^{\prime}$ such that $\operatorname{dim} V=\operatorname{dim} V^{\prime}$ and the restriction $\left.f\right|_{V}: V \rightarrow V^{\prime}$ is birational. We call $V^{\prime}$ the birational image of $V$ by $f$ and denote $V^{\prime}:=f_{*} V$.

Note that the composition of small birational maps that are isomorphic at the generic point of $V$ and its birational images is also isomorphic at the generic point of $V$.

Theorem 3.2. (cf. [4, p. 8]) Let $(X, B)$ be a projective klt pair such that $K+B$ is big. Let $\varphi: X \rightarrow X^{\prime}$ be a divisorial contraction or a log flip of $(K+B)$. Then the following hold:

(1) $\varphi$ is isomorphic at the generic point of all the irreducible components not contained in $\mathbf{B}_{-}(K+B)$.

(2) Let $V$ be an irreducible component of $\mathbf{B}_{+}(K+B)$ not contained in $\mathbf{B}_{-}(K+$ $B)$. Then the birational image $V^{\prime}$ of $V$ by $f$ is an irreducible component of $\mathbf{B}_{+}\left(K_{X^{\prime}}+B_{X^{\prime}}\right)$ not contained in $\mathbf{B}_{-}(K+B)$.

(3) The association in (2) of the irreducible components defined by $f_{*}$ is bijective for the divisorial components.

See $[4$, p. 8$]$ for essentially the same statements. Note that if $(X, B)$ is a projective klt pair such that $K+B$ is big, then we can obtain a log minimal model of $(X, B)$ by running the LMMP (with scaling) which consists of finitely many divisorial contractions and log flips [2]. In particular, we have the following

Corollary 3.1. Under the same conditions as in Theorem 3.2, if $f: X \rightarrow Y$ is a log minimal model of $(X, B)$ obtained by running the LMMP (with scaling), then $f$ is isomorphic at the generic point of any irreducible component of $\mathbf{B}_{+}(K+B)$ not contained in $\mathbf{B}_{-}(K+B)$ and its birational image is an irreducible component of $\mathbf{B}_{+}\left(K_{Y}+B_{Y}\right)$. Furthermore, such association of components is bijective for the divisorial components.

Proof of Theorem 3.2. (1) It is well known that the exceptional divisor of a divisorial contraction and the flipping locus of a log flip are contained in $\mathbf{B}_{-}(K+B)$. Thus such map $\varphi$ is generically isomorphic on the subvarieties not contained in $\mathbf{B}_{-}(K+B)$.

(2) Let $V$ be an irreducible component of $\mathbf{B}_{+}(K+B)$ not contained in $\mathbf{B}_{-}(K+B)$. By (1), $\varphi$ is isomorphic at the generic point of $V$ and let $V^{\prime}:=f_{*} V$ be its birational image. By Proposition 2.1, there exists a divisorial valuation $\sigma$ of $X$ such that $\operatorname{Cent}_{X} \sigma=V$ and $\sigma_{\text {num }}(K+B)=0$. 
Let $\varphi: X \rightarrow X^{\prime}$ be an extremal divisorial contraction of $(X, B)$ contracting a prime divisor $E$. Then we have $K+B=\varphi^{*}\left(K_{X^{\prime}}+B_{X^{\prime}}\right)+t E$ for some $t>0$. Thus by Lemma $2.2, \sigma_{\text {num }}\left(K_{X^{\prime}}+B_{X^{\prime}}\right)=0$ and since we may assume that $\operatorname{Cent}_{X^{\prime}} \sigma=V^{\prime}, V^{\prime}$ is not contained in $\mathbf{B}_{-}\left(K_{X^{\prime}}+B_{X^{\prime}}\right)$. Furthermore, by Lemma $2.1, V^{\prime}$ is an irreducible component of $\mathbf{B}_{+}\left(K_{X^{\prime}}+B_{X^{\prime}}\right)$.

Now let $\varphi: X \rightarrow X^{\prime}$ be a log flip of $(X, B)$ and let $p: W \rightarrow X, q: W \rightarrow$ $X^{\prime}$ be common log resolutions of $(X, B)$ and $\left(X^{\prime}, B_{X^{\prime}}\right)$, respectively. Then we have $p^{*}(K+B)=q^{*}\left(K_{X^{\prime}}+B_{X^{\prime}}\right)+F$ for some $p, q$-exceptional effective divisor $F$. Thus by Lemma 2.2 ,

$$
\sigma_{\text {num }}(K+B)=\sigma_{\text {num }}\left(K_{X^{\prime}}+B_{X^{\prime}}\right)+\sigma(F),
$$

for any divisorial valuation $\sigma$ of $X$. Arguing the same way as above, since $\sigma_{\text {num }}(K+$ $B)=0$ if Cent $_{X} \sigma=V$, we have $V^{\prime} \nsubseteq \mathbf{B}_{-}\left(K_{X^{\prime}}+B_{X^{\prime}}\right)$. Now suppose that $V^{\prime} \nsubseteq$ $\mathbf{B}_{+}\left(K_{X^{\prime}}+B_{X^{\prime}}\right)$. Since $\mathbf{B}_{+}\left(K_{X^{\prime}}+B_{X^{\prime}}\right)=\mathbf{B}_{-}\left(K_{X^{\prime}}+B_{X^{\prime}}-H^{\prime}\right)$ for a sufficiently small ample divisor $H^{\prime}$ on $X^{\prime}$, we have $\sigma_{\text {num }}\left(K_{X^{\prime}}+B_{X^{\prime}}-H^{\prime}\right)=0$ by Corollary 2.1 . Perturbing the equality above by the ample divisors $H^{\prime}$ and $H:=\chi_{*}^{-1} H^{\prime}$, we have

$$
\sigma_{\text {num }}(K+B-H)=\sigma_{\text {num }}\left(K_{X^{\prime}}+B_{X^{\prime}}-H^{\prime}\right)+\sigma\left(F^{\prime}\right)
$$

where $F^{\prime}$ is an effective $p, q$-exceptional divisor such that $\operatorname{Supp} F^{\prime}=\operatorname{Supp} F$. Since $H^{\prime}$ can be chosen arbitrarily small, we may assume that $\mathbf{B}_{+}(K+B)=\mathbf{B}_{-}(K+B-H)$ and since $V \subseteq \mathbf{B}_{+}(K+B)$, we have $\sigma_{\text {num }}(K+B-H)>0$ by Corollary 2.1. Thus $\sigma\left(F^{\prime}\right)>0$ which implies $V=\operatorname{Cent}_{X} \sigma \subseteq \operatorname{Cent}_{X} F^{\prime}$. Note that since Cent ${ }_{X} F^{\prime}$ is contained in the flipping locus of $\varphi$ [10, Lemma 3.4] (or [12, Lemma 3.38]), $V$ is contained in the flipping locus of $\varphi$ and $V \subseteq \mathbf{B}_{-}(K+B)$ by (1). However, this contradicts our given condition. Thus $V^{\prime} \subseteq \mathbf{B}_{+}\left(K_{X^{\prime}}+B_{X^{\prime}}\right)$. Suppose that $V^{\prime}$ is not an irreducible component of $\mathbf{B}_{+}\left(K_{X^{\prime}}+B_{X^{\prime}}\right)$, i.e., there exists an irreducible component $V^{\prime \prime} \subseteq \mathbf{B}_{+}\left(K_{X^{\prime}}+B_{X^{\prime}}\right)$ such that $V^{\prime} \subsetneq V^{\prime \prime}$. Then by arguing as above, for a divisorial valuation $\lambda$ such that Cent $_{X^{\prime}} \lambda=V^{\prime \prime}$ we have $\lambda_{\text {num }}\left(K_{X^{\prime}}+B_{X^{\prime}}-H^{\prime}\right)>0$ for a sufficiently small ample divisor $H^{\prime}$ on $X^{\prime}$. This would imply as above that $\lambda_{\text {num }}(K+B-H)>0$ for a sufficiently small ample divisor $H$ and $V_{X}^{\prime \prime}:=\operatorname{Cent}_{X} \lambda \subseteq \mathbf{B}_{-}(K+B-H)=$ $\mathbf{B}_{+}(K+B)$. Clearly, $V \subsetneq V_{X}^{\prime \prime}$ and this contradicts the given condition that $V$ is a (maximal) irreducible component of $\mathbf{B}_{+}(K+B)$.

(3) Let $D^{\prime}$ be a divisorial component of $\mathbf{B}_{+}\left(K_{X^{\prime}}+B_{X^{\prime}}\right)$ not contained in $\mathbf{B}_{-}\left(K_{X^{\prime}}+\right.$ $\left.B_{X^{\prime}}\right)$. By $(2)$, we need to show that $D^{\prime}$ is a birational image of some divisorial component of $\mathbf{B}_{+}(K+B)$ not contained in $\mathbf{B}_{-}(K+B)$.

Suppose that $\varphi: X \rightarrow X^{\prime}$ is a divisorial contraction with the $\varphi$-exceptional prime divisor $E$. Then we have $K+B=\varphi^{*}\left(K_{X^{\prime}}+B_{X^{\prime}}\right)+t E$ for some $t>0$. Clearly, $D:=\varphi_{*}^{-1} D^{\prime}$ is not $\varphi$-exceptional. Thus by Lemma $2.1, E$ is an irreducible component of $\mathbf{B}_{+}(K+B)$. Since $D \neq E$, we have $\sigma(E)=0$ for the divisorial valuation $\sigma$ such that $\operatorname{Cent}_{X} \sigma=D$. Thus by Lemma $2.2, \sigma_{\text {num }}(K+B)=\sigma_{\text {num }}\left(K_{X^{\prime}}+B_{X^{\prime}}\right)=0$ and Proposition 2.1 implies that $D \nsubseteq \mathbf{B}_{-}(K+B)$.

Now suppose that $\varphi: X \rightarrow X^{\prime}$ is a log flip of $(X, B)$. Let $W$ be a common resolution of $(X, B)$ and $\left(X^{\prime}, B_{X^{\prime}}\right)$ with $p: W \rightarrow X$ and $q: W \rightarrow X^{\prime}$. Then we have $p^{*}(K+B)=q^{*}\left(K_{X^{\prime}}+B_{X^{\prime}}\right)+G$ for some $p$, $q$-exceptional effective divisor $G$. By Lemma 2.2,

$$
p^{-1}\left(\mathbf{B}_{+}(K+B)\right) \cup \operatorname{Exc}(p)=q^{-1}\left(\mathbf{B}_{+}\left(K_{X^{\prime}}+B_{X^{\prime}}\right)\right) \cup \operatorname{Exc}(q) .
$$


Clearly, $D_{W}:=q_{*}^{-1} D^{\prime}$ is not $p$-exceptional and thus $D=p\left(D_{W}\right)$ is an irreducible component of $\mathbf{B}_{+}(K+B)$. Note that the multiplicity of $G$ is positive only at the prime divisors whose centers belong to the flipping locus [10, Lemma 3.4] (or [12, Lemma $3.38]$ ). Therefore if $\sigma$ is a divisorial valuation of $X$ such that $\operatorname{Cent}_{X^{\prime}} \sigma=D^{\prime}$, then we have $\sigma(G)=0$. Since $D^{\prime} \nsubseteq \mathbf{B}_{-}\left(K_{X^{\prime}}+B_{X^{\prime}}\right), \sigma_{\text {num }}\left(K_{X^{\prime}}+B_{X^{\prime}}\right)=0$ and Lemma 2.2 implies that $D=\operatorname{Cent}_{X} \sigma \nsubseteq \mathbf{B}_{-}(K+B)$.

Theorem 3.3. Let $(X, B)$ be a projective klt pair such that $K+B$ is big. Then the divisorial components of $\mathbf{B}_{+}(K+B)$ are precisely the divisors on $X$ that are exceptional on the log canonical model $X_{\text {can }}$ of $(X, B)$. Furthermore, if $f: X \rightarrow Y$ is a log minimal model of $(X, B)$ obtained by running the LMMP (with scaling), then $\mathbf{B}_{+}\left(K_{Y}+B_{Y}\right)$ is precisely the exceptional locus over $X_{\text {can }}$.

Proof. Let $E$ be a divisorial component of $\mathbf{B}_{+}(K+B)$.

If $E \subseteq \mathbf{B}_{-}(K+B)$, then by Theorem 3.1, $E$ is exceptional on a log minimal model $f: X \rightarrow Y$ of $(X, B)$. Thus $E$ is also exceptional on the $\log$ canonical model $X_{\text {can }}$.

Let $E \nsubseteq \mathbf{B}_{-}(K+B)$. If $f: X \rightarrow Y$ is a $\log$ minimal model of $(X, B)$, then by Theorem $3.1 E$ is not contracted by $f$. By (2) of Theorem $3.2, E_{Y}:=f_{*} E$ is a divisorial component of $\mathbf{B}_{+}\left(K_{Y}+B_{Y}\right)$. Now it is easy to see that by Lemma 2.1, $E_{Y} \subseteq \operatorname{Exc}(g)$ where $g: Y \rightarrow X_{\text {can }}$ is the log canonical contraction of $\left(Y, B_{Y}\right)$ to the $\log$ canonical model $X_{\text {can }}$. Therefore $E$ is exceptional on $X_{\text {can }}$.

Conversely, let $E$ be a prime divisor on $X$ which is exceptional on $X_{\text {can }}$. If $E$ is exceptional on a $\log$ minimal model $f: X \rightarrow Y$ of $(X, B)$, then by Theorem 3.1 $E$ is a divisorial component of $\mathbf{B}_{-}(K+B)$ and also of $\mathbf{B}_{+}(K+B)$. Thus we may assume that $E$ is not exceptional on any $\log$ minimal model $f: X \rightarrow Y$ of $(X, B)$. Let $E_{Y}:=f_{*} E$ and $g: Y \rightarrow X_{\text {can }}$ be the log canonical contraction of $\left(Y, B_{Y}\right)$. Then $E_{Y}$ is $g$-exceptional. Since $K_{Y}+B_{Y}=g^{*}\left(K_{X_{\text {can }}}+B_{X_{\text {can }}}\right)$, by Lemma $2.1 E_{Y}$ is a divisorial component of $\mathbf{B}_{+}\left(K_{Y}+B_{Y}\right)$. Thus by (3) of Theorem 3.2, $E$ is a divisorial component of $\mathbf{B}_{+}(K+B)$.

The equality $K_{Y}+B_{Y}=g^{*}\left(K_{X_{\text {can }}}+B_{X_{\text {can }}}\right)$ with Lemma 2.1 proves the last statement.

\section{Duality of cones}

In this section, we prove Theorem 1.1 and Corollary 1.1, thereby giving some partial affirmative answers to Problem 1.1.

The following definitions are inspired by [17].

Definition 4.1. Let $X$ be a $\mathbb{Q}$-factorial projective variety of dimension $d$ and $k$ be an integer such that $0 \leq k \leq d-1$.

(1) A curve $C$ on $X$ is called a $m o v^{k}$ (movable in codimension $k$ )-curve if it belongs to a family of curves covering a subvariety $V \subseteq X$ of codimension $k$. In this case, we say that $C$ is movable in $V$. A mov ${ }^{0}$-curve $C$ is often called a movable curve.

(2) Let $f: X \rightarrow X^{\prime}$ be a small birational map such that $f^{-1}$ is isomorphic at the generic point of $V^{\prime} \subseteq X^{\prime}$ of codimension $k$. A mov ${ }^{k}$-curve $C^{\prime}$ on $X^{\prime}$ which is movable in $V^{\prime}$ is called a $b$-mov $v^{k}$ (birationally movable in codimension $k)$-curve of $X$. 
As explained in Introduction, if $f: X \rightarrow X^{\prime}$ is a small birational map of $\mathbb{Q}$-factorial varieties, then $f$ not only induces an isomorphism $\mathrm{N}^{1}(X) \cong \mathrm{N}^{1}\left(X^{\prime}\right)$ but also the isomorphism $\mathrm{N}_{1}(X) \cong \mathrm{N}_{1}\left(X^{\prime}\right)$. Therefore, a b-mov ${ }^{k}$-curve $C$ of $X$ defines a class $\alpha$ in $\mathrm{N}_{1}(X)$ via the above isomorphism even though $\alpha$ may not represent a well defined curve on $X$ (see also the numerical pull-back of [1]). Therefore, we may (and will) treat b-mov ${ }^{k}$-curves of $X$ as classes in $\mathrm{N}_{1}(X)$.

Definition 4.2. Let $X$ be a $\mathbb{Q}$-factorial projective variety of dimension $d$ and $k$ be an integer such that $0 \leq k \leq d-1$. We define

(1) $\mathrm{NM}^{k}(X)$ as the convex cone in $\mathrm{N}_{1}(X)$ spanned by the mov ${ }^{k}$-curves on $X$, and

(2) $\mathrm{bNM}^{k}(X)$ as the convex cone in $\mathrm{N}_{1}(X)$ spanned by the b-mov ${ }^{k}$-curves of $X$.

Clearly, we have the following inclusions:

$$
\begin{aligned}
\overline{\mathrm{NM}}^{k}(X) & \subseteq \overline{\mathrm{NM}}^{k+1}(X), \\
\overline{\mathrm{bNM}}^{k}(X) & \subseteq \overline{\mathrm{bNM}}^{k+1}(X), \quad \text { and } \\
\overline{\mathrm{NM}}^{k}(X) & \subseteq \overline{\mathrm{bNM}}^{k}(X) .
\end{aligned}
$$

Note also that $\overline{\mathrm{NM}}^{0}(X)=\overline{\mathrm{bNM}}^{0}(X)$ is the cone of movable curves and $\overline{\mathrm{bNM}}^{d-1}(X)=\overline{\mathrm{NE}}(X)$ is the cone of effective curves (often called the Mori cone). Let $f: X \rightarrow X^{\prime}$ be a small birational map between $\mathbb{Q}$-factorial varieties. We define $\overline{\mathrm{NM}}^{k}\left(X, X^{\prime}\right)$ as the image of the cone $\overline{\mathrm{NM}}^{k}\left(X^{\prime}\right) \subseteq \mathrm{N}_{1}\left(X^{\prime}\right)$ in $\mathrm{N}_{1}(X)$ via the isomorphism $\mathrm{N}_{1}\left(X^{\prime}\right) \cong \mathrm{N}_{1}(X)[17]$. Then it is easy to see that

$$
\overline{\mathrm{bNM}}^{k}(X)=\overline{\sum_{X \rightarrow X^{\prime}} \overline{\mathrm{NM}}^{k}\left(X, X^{\prime}\right)},
$$

where the sum is taken over all $\mathbb{Q}$-factorial small birational modifications $X^{\prime}$ of $X$.

Definition 4.3. Let $X$ be a projective variety of dimension $d$ and $k$ be an integer such that $1 \leq k \leq d$. We define

(1) $\operatorname{Amp}_{k}(X)$ as the convex cone in $\mathrm{N}^{1}(X)$ spanned by the divisors $D$ ample in dimension $k$. i.e., $\operatorname{dim} \mathbf{B}_{+}(D)<k$,

(2) $\operatorname{Nef}_{k}(X)$ as the convex cone in $\mathrm{N}^{1}(X)$ spanned by the divisors $D$ nef in dimension $k$. i.e., $\operatorname{dim} \mathbf{B}_{-}(D)<k$, and

(3) $\operatorname{SB}_{k}(X)$ as the convex cone in $\mathrm{N}^{1}(X)$ spanned by the $\mathbb{Q}$-divisors $D$ such that the stable base loci $\mathbf{B}(D)$ do not contain any $k$-dimensional subvarieties.

Clearly, we have the following inclusions:

$$
\begin{aligned}
\operatorname{Amp}_{k}(X) & \subseteq \operatorname{Amp}_{k+1}(X), \\
\operatorname{Nef}_{k}(X) & \subseteq \operatorname{Nef}_{k+1}(X), \quad \text { and } \\
\operatorname{Amp}_{k}(X) & \subseteq \operatorname{SB}_{k}(X) \subseteq \operatorname{Nef}_{k}(X) .
\end{aligned}
$$

Note also that $\operatorname{Amp}_{1}(X)=\operatorname{Amp}(X)$ is the ample cone, $\operatorname{Nef}_{1}(X)=\operatorname{Nef}(X)$ the nef cone, $\operatorname{Amp}_{d}(X)=\operatorname{Big}(X)$ the cone of big divisors, and $\operatorname{Nef}_{d}(X)=\overline{\mathrm{Eff}}(X)$ the pseudo-effective cone. The cone $\overline{\operatorname{Amp}}_{d-1}(X)=\operatorname{Nef}_{d-1}(X)=\overline{\operatorname{Mob}}(X)$ is the mobile cone $[6]$. 
Remark 4.1. It is easy to see that $\overline{\operatorname{Amp}}_{k}(X) \subseteq \overline{\mathrm{NM}}^{d-k}(X)^{\vee}$. (For a divisor $D \in$ $\operatorname{Amp}_{k}(X)$ and a mov ${ }^{d-k}$-curve $C$, we have $D \cdot C \geq 0$ because we may assume that $C$ is disjoint from the base locus of $D$.) It is important to note that as pointed out in [17, Example 1] the inclusion $\overline{\operatorname{Amp}}_{k}(X) \subsetneq \overline{\mathrm{NM}}^{d-k}(X)^{\vee}$ is strict in general. Thus considering the curves only on $X$ is not enough in the duality of Problem 1.1.

The following is well known for $k=1, d=\operatorname{dim} X$.

Theorem 4.1. Let $\operatorname{Amp}_{k}(X), \operatorname{Nef}_{k}(X)(k=1,2, \ldots, d=\operatorname{dim} X)$ be the cones in $\mathrm{N}^{1}(X)$ as defined above.

(1) Let $D \in \overline{\operatorname{Amp}}_{k}(X)$. Then $D+A \in \operatorname{Amp}_{k}(X)$ for any ample divisor $A$.

(2) The cone $\operatorname{Amp}_{k}(X)$ is open and

$$
\overline{\operatorname{Amp}}_{k}(X)=\overline{\mathrm{SB}}_{k}(X)=\operatorname{Nef}_{k}(X) \text {. }
$$

In particular, the cone $\operatorname{Nef}_{k}(X)$ is closed and $\operatorname{Int} \operatorname{Nef}_{k}(X)=\operatorname{Amp}_{k}(X)$.

Proof. (1) Since $D \in \overline{\operatorname{Amp}}_{k}(X)$, there exists a sequence $D_{i} \in \operatorname{Amp}_{k}(X)$ such that $D_{i} \rightarrow D$ as $i \rightarrow \infty$. For a fixed ample divisor $A$, by taking $i$ sufficiently large, we may assume that $A-\left(D_{i}-D\right)$ is ample for all $i$. Thus $\mathbf{B}_{+}\left(D_{i}\right) \supseteq \mathbf{B}_{+}\left(D_{i}+A-\left(D_{i}-D\right)\right)=$ $\mathbf{B}_{+}(D+A)$ and $D+A \in \operatorname{Amp}_{k}(X)$.

(2) The openness of the cone $\operatorname{Amp}_{k}(X)$ follows from [8, Corollary 1.6]: there exists a small open neighborhood $N$ of $D$ such that for any $D^{\prime} \in N, \mathbf{B}_{+}\left(D^{\prime}\right) \subseteq \mathbf{B}_{+}(D)$.

It is enough to prove $\overline{\operatorname{Amp}}_{k}(X)=\operatorname{Nef}_{k}(X)$. To prove the inclusion $\overline{\operatorname{Amp}}_{k}(X) \subseteq$ $\operatorname{Nef}_{k}(X)$, let $D \in \overline{\operatorname{Amp}}_{k}(X)$. If $D \notin \operatorname{Nef}_{k}(X)$, then there exists a $k$-dimensional subvariety $E \subseteq X$ such that $E \subseteq \mathbf{B}_{-}(D)$. By remark 2.1, $E \subseteq \mathbf{B}_{+}(D+A)$ for some ample divisor $A$, but it is a contradiction to (1). The inclusion $\overline{\operatorname{Amp}}_{k}(X) \supseteq$ $\operatorname{Nef}_{k}(X)$ can be seen as follows. Let $D \in \operatorname{Nef}_{k}(X)$. Then for a fixed ample divisor $A$, $\left\{D+\frac{1}{i} A\right\}_{i \in \mathbb{N}}$ is a sequence in $\operatorname{Amp}_{k}(X)$ since $\mathbf{B}_{+}\left(D+\frac{1}{i} A\right) \subseteq \mathbf{B}_{-}(D)$. Thus the limit of the sequence must belong to $\overline{\operatorname{Amp}}_{k}(X)$, i.e., $D \in \overline{\operatorname{Amp}}_{k}(X)$.

Corollary 4.1. Let $D \in \partial \overline{\operatorname{Amp}}_{k}(X)$ where $1 \leq k \leq d-1$. Then there exists a k-dimensional subvariety $V$ which is contained in $\mathbf{B}_{+}(D)$. If additionally $D \in$ $\operatorname{Amp}_{k+1}(X)$, then $V$ is an irreducible component of $\mathbf{B}_{+}(D)$.

Proof. By Theorem 4.1, $D \notin \operatorname{Amp}_{k}(X)$. Thus there exists a $k$-dimensional subvariety $V$ contained in $\mathbf{B}_{+}(D)$. If $D \in \operatorname{Amp}_{k+1}(X)$, then there are no $(k+1)$-dimensional subvarieties $V^{\prime} \subseteq \mathbf{B}_{+}(D)$. Thus $V$ is an irreducible component.

We first recall the well known duality.

Theorem 4.2. The following duality hold:

(1) (the case $k=1): \overline{\operatorname{Amp}}_{1}(X)^{\vee}=\overline{\mathrm{NM}}^{d-1}(X)$ (= $\overline{\mathrm{NE}}(X)$, the Mori cone),

(2) (the case $k=d): \overline{\operatorname{Amp}}_{d}(X)^{\vee}=\overline{\mathrm{NM}}^{0}(X)(=\overline{\mathrm{NM}}(X)$, the cone of movable curves), and

Proof. (1) See [13, Proposition 1.4.28].

(2) It is the main result of [5] for smooth varieties. The result also holds for $\mathbb{Q}$ factorial varieties [13]. 
The following theorem tells us that the duality of Problem 1.1 for general $k$ holds at least in some portion of the $(K+B)$-negative part when $(X, B)$ is klt. We fix a notation:

$$
\overline{\mathrm{NE}}(X)_{K+B \geq 0}:=\overline{\mathrm{NE}}(X) \cap\left\{C \in \mathrm{N}^{1}(X) \mid C \cdot(K+B) \geq 0\right\} .
$$

Theorem 4.3. Let $(X, B)$ be a $\mathbb{Q}$-factorial projective klt pair. Let $1 \leq k \leq d=\operatorname{dim} X$. Then the following holds:

$$
\overline{\mathrm{NE}}(X)_{K+B \geq 0}+\overline{\operatorname{Amp}}_{k}(X)^{\vee}=\overline{\mathrm{NE}}(X)_{K+B \geq 0}+\overline{\mathrm{bNM}}^{d-k}(X) .
$$

The proof of this theorem is essentially the same as that of [6, Theorem 1.1] where the case $k=d-1$ is proved. For general $k$, we only need to take care of the effect of the LMMP on the components of the base loci $\mathbf{B}_{+}(K+B)$ and $\mathbf{B}_{-}(K+B)$ using Corollary 3.1.

Proof of Theorem 4.3. We will prove the following equivalent dual statement by reverse induction on $k$ arguing as in [5, Theorem 2.2]:

$$
\begin{aligned}
\operatorname{Dual}(k): & \overline{\operatorname{Amp}}_{k}(X) \cap P=\overline{\mathrm{bNM}}^{d-k}(X)^{\vee} \cap P \\
& \text { where } P=\operatorname{Nef}(X)+\mathbb{R}_{\geq 0} \cdot[K+B] .
\end{aligned}
$$

First of all, $\operatorname{Dual}(d)$ holds by Theorem 4.2. Assume that $\operatorname{Dual}(k+1)$ holds for $k+1 \leq d$ and we claim that $\operatorname{Dual}(k)$ holds as well.

STEP 1: We first prove that the inclusion $\subseteq$ holds in $\operatorname{Dual}(k)$. It is enough to prove $\overline{\operatorname{Amp}}_{k}(X) \cap \operatorname{Int} P \subseteq \overline{\mathrm{bNM}}^{d-k}(X)^{\vee} \cap \operatorname{Int} P$. Any divisor $D \in \overline{\operatorname{Amp}}_{k}(X) \cap \operatorname{Int} P$ has the form $D \equiv K+B+H$ for some ample divisor $H$. We may assume that $(X, B+H)$ is klt and since $K+B+H \in \overline{\operatorname{Amp}}_{k}(X)$, the LMMP $f: X \rightarrow X^{\prime}$ with scaling on the pair $(X, B+H)$ is an isomorphism in codimension $d-k$. The divisor $K_{X^{\prime}}+B_{X^{\prime}}+H_{X^{\prime}}$ is nef, hence belongs to $\overline{\mathrm{NE}}\left(X^{\prime}\right)^{\vee} \subseteq \overline{\mathrm{bNM}}^{d-k}\left(X^{\prime}\right)^{\vee}$. Thus we also have $D \equiv K+B+H \in$ $\overline{\mathrm{bNM}}^{d-k}(X)^{\vee}$.

STEP 2: Now suppose that the strict inequality $\subsetneq$ holds in $\operatorname{Dual}(k)$. Note that since $\overline{\mathrm{bNM}}^{d-k}(X) \supseteq \overline{\mathrm{bNM}}^{d-(k+1)}(X)$, we have by dualizing

$$
\overline{\operatorname{Amp}}_{k}(X) \cap P \subsetneq \overline{\mathrm{bNM}}^{d-k}(X)^{\vee} \cap P \subseteq \overline{\mathrm{bNM}}^{d-(k+1)}(X)^{\vee} \cap P=\overline{\operatorname{Amp}}_{k+1}(X) \cap P .
$$

The last equality holds by the induction hypothesis. Note also that $\overline{\mathrm{bNM}}^{d-k}(X)^{\vee}=$ $\cap \overline{\mathrm{NM}}^{d-k}\left(X, X^{\prime}\right)^{\vee}$ where the intersection is taken over all $\mathbb{Q}$-factorial $X^{\prime}$ isomorphic to $X$ in codimension 1. Thus there exists a divisor $D \in \operatorname{Amp}_{k+1}(X)$ such that $D \in$ $\partial \overline{\operatorname{Amp}}_{k}(X) \cap \operatorname{Int} P$ and $D \in \operatorname{Int}\left(\overline{\mathrm{NM}}^{d-k}\left(X, X^{\prime}\right)^{\vee}\right)$ for any $\mathbb{Q}$-factorial $X^{\prime}$ isomorphic

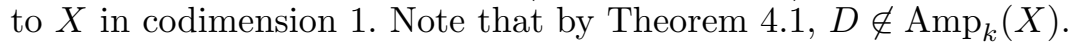

STEP 3: There exists an ample divisor $H$ such that $r D \equiv K+B+H$ for some $r>0$. By rescaling $D$, we may assume that $r=1$ and we may assume that $(X, B+H)$ is klt. 
By running the LMMP on $(X, B+H)$ with scaling, we obtain a log minimal model $f: X \rightarrow Y$ of $(X, B+H)$. Note that the map $f$ is small because $D \equiv K+B+H \in$ $\overline{\operatorname{Amp}}_{k}(X) \subseteq \overline{\operatorname{Mob}}(X)$ with $k \leq d-1$ (Theorem 3.1) and that since $D \in \partial \overline{\operatorname{Amp}}_{k}(X)$ and $D \in \operatorname{Amp}_{k+1}(X)$, there exists a $k$-dimensional irreducible component $V$ of $\mathbf{B}_{+}(D)$ not contained in $\mathbf{B}_{-}(D)$ by Corollary 4.1. Furthermore, by Theorem 3.2, the map $f$ is isomorphic at the generic point of $V$ and its birational image $V^{\prime}=f_{*} V$ is also a $k$-dimensional irreducible component of $\mathbf{B}_{+}\left(D_{Y}\right)$.

STEP 4: Since $D \in \operatorname{Int}\left(\overline{\mathrm{NM}}^{d-k}(X, Y)^{\vee}\right) \cong \operatorname{Int}\left(\overline{\mathrm{NM}}^{d-k}(Y)^{\vee}\right)$, we have $D_{Y}:=f_{*} D \in$ $\operatorname{Int}\left(\overline{\mathrm{NM}}^{d-k}(Y)^{\vee}\right)$. Thus there exists a small ample divisor $H$ such that $\left(D_{Y}-H\right) \cdot C>0$ for any mov ${ }^{d-k}$-curve $C$ on $Y$. Let $C=V^{\prime} \cdot\left(D_{Y}+\lambda H\right)^{k-1}$ be a mov ${ }^{d-k}$-curve on $Y$ movable in $V^{\prime}$. Applying this curve $C$ to $D_{Y} \cdot C>H \cdot C$, we obtain

$$
\begin{aligned}
\left(D_{Y}+\lambda H\right)^{k} \cdot V^{\prime} & >D_{Y} \cdot\left(D_{Y}+\lambda H\right)^{k-1} \cdot V^{\prime} \\
& >H \cdot\left(D_{Y}+\lambda H\right)^{k-1} \cdot V^{\prime} \\
& \geq\left(H^{k} \cdot V^{\prime}\right)^{\frac{1}{k}} \cdot\left(\left(D_{Y}+\lambda H\right)^{k} \cdot V^{\prime}\right)^{\frac{k-1}{k}}
\end{aligned}
$$

where the last inequality holds by Khovanskii-Teissier inequality [13, Theorem 1.6.1]. Simplifying the inequality yields

$$
\left(D_{Y}+\lambda H\right)^{k} \cdot V^{\prime} \geq H^{k} \cdot V^{\prime}
$$

Since $H$ is ample, $H^{k} \cdot V^{\prime}>0$. However, if we take the limit $\lambda \rightarrow 0,\left(D_{Y}+\lambda H\right)^{k}$. $V^{\prime} \rightarrow D_{Y}^{k} \cdot V^{\prime}$ and $D_{Y}^{k} \cdot V^{\prime}=0$ by Nakamaye's theorem (Theorem 2.1). It is a contradiction.

We prove that the duality of Problem 1.1 holds for the following type of varieties.

Definition 4.4. [18, Lemma-Definition 2.8] A normal projective variety $X$ is said to be $F T$ if one of the following equivalent conditions holds:

(1) there exists a $\mathbb{Q}$-boundary divisor $B$ on $X$ such that $(X, B)$ is a klt and $-(K+B)$ is ample;

(2) there exists a $\mathbb{Q}$-boundary divisor $B$ on $X$ such that $(X, B)$ is klt, $K+B \sim_{\mathbb{Q}} 0$ and the divisors in the support of $B$ generate $\mathrm{N}^{1}(X)$.

Corollary 4.2. The duality of Problem 1.1 holds for $\mathbb{Q}$-factorial FT varieties of arbitrary dimension $d$ for all integers $k$ such $1 \leq k \leq d$.

Proof. There exists a $\mathbb{Q}$-boundary divisor $B$ such that $(X, B)$ is a klt, $K+B \equiv 0$, and the divisors in the support of $B$ generate $\mathrm{N}^{1}(X)$. Take an effective ample divisor $H$ supported in $\operatorname{Supp} B$. Then for sufficiently small $\varepsilon>0, B-\varepsilon H$ is effective and $(X, B-$ $\varepsilon H)$ is klt. Furthermore, $-(K+B-\varepsilon H)$ is ample. Since the cone $N E(X) \backslash\{0\}$ is $(K+$ $B-\varepsilon H)$-negative, the duality $\overline{\operatorname{Amp}}_{k}(X)=\overline{\mathrm{bNM}}^{d-k}(X)^{\vee}$ holds by Theorem 4.3.

We recall that that the $D$-MMP for any divisor $D$ works on a Mori dream space [9].

Corollary 4.3. The duality of Problem 1.1 holds for a Mori dream space $X$.

Proof. The statement follows if for all divisors $D$ on $X$, the cones $\overline{\operatorname{Amp}}_{k}(X)$ and $\overline{\mathrm{bNM}}^{d-k}(X)^{\vee}$ coincide inside the cone $P=\overline{\mathrm{NE}}(X)+\mathbb{R}_{\geq 0}[D]$ (cf. $\operatorname{Dual}(k)$ in the proof of Theorem 4.3). 
As in Step 1 of Theorem 4.3, the validity of the $D$-MMP on a Mori dream space for any divisor $D$ implies that $\overline{\operatorname{Amp}}_{k}(X) \subseteq \overline{\mathrm{bNM}}^{d-k}(X)$ in $P$.

Suppose that there exists a divisor $D$ such that the inclusion is strict. Then after replacing $K+B$ by $D$ in the proof of Theorem 4.3 , we can carry out the proof of the statement as in the proof of Theorem 4.3 to obtain a contradiction.

It is known that the cone $\overline{\operatorname{Amp}}_{k}(X)$ for a $d$-dimensional FT variety $X$ is rational polyhedral for $k=1, d-1$ and $d$ [19]. More generally, we may ask the following.

Question 4.4. Are the cones $\overline{\operatorname{Amp}}_{k}(X)$ for a $\mathbb{Q}$-factorial FT variety or a Mori dream space $X$ rational polyhedral?

\section{Acknowledgment}

The author would like to thank the referee for pointing out errors in the preliminary version of the paper and for useful comments and suggestions.

\section{References}

[1] C. Araujo, The cone of pseudo-effective divisors of log varieties after Batyrev. Math. Z. 264(1) (2010), 179-193.

[2] C. Birkar, P. Cascini, C. Hacon and J. McKernan, Existence of minimal models for varieties of log general type. J. Amer. Math. Soc. 23(2) (2010), 405-468.

[3] S. Boucksom, Divisorial Zariski decompositions on compact complex manifolds. Ann. Sci. École Norm. Sup. (4) 37(1) (2004), 45-76.

[4] S. Boucksom, A. Broustet and G. Pacienza, Uniruledness of stable base loci of adjoint linear systems with and without Mori Theory, arXiv:0902.1142v2.

[5] S. Boucksom, J.P. Demailly, M. Paun and T. Peternell, The pseudo-effective cone of a compact Kähler manifold and varieties of negative Kodaira dimension, arXiv:math/0405285v1, to appear in J. Algebra. Geom.

[6] S. Choi, On the dual of the mobile cone, arXiv:1011.4646v3, to appear in Math. Z., doi: 10.1007/s00209-011-0922-7.

[7] L. Ein, R. Lazarsfeld, M. Mustată, M. Nakamaye and M. Popa, Restricted volumes and base loci of linear series, Amer. J. Math. 131(3) (2009), 607-651.

[8] L. Ein, R. Lazarsfeld, M. Mustată, M. Nakamaye and M. Popa, Asymptotic invariants of base loci, Ann. Inst. Fourier 56(6) (2006), 1701-1734.

[9] Y. Hu and S. Keel, Mori dream spaces and GIT, Michigan Math. J. 48 (2000), 331-348.

[10] V.A. Iskovskikh and V.V. Shokurov, Birational models and flips, Russ. Math. Surv. 60 (2005), $27-94$.

[11] Y. Kawamata, Remarks on the cone of divisors, in Classification of Algebraic Varieties, EMS Series of Congress Reports. European Mathematical Society (EMS), 2011.

[12] J. Kollár and S. Mori, Birational geometry of algebraic varieties, Cambridge University Press, $1998,134$.

[13] R. Lazarsfeld, Positivity in algebraic geometry I, 48. xviii+385pp. Springer-Verlag, Berlin, 2004.

[14] R. Lazarsfeld, Positivity in algebraic geometry II, 49. xviii+387pp. Springer-Verlag, Berlin, 2004.

[15] M. Nakamaye, Stable base loci of linear series, Math. Ann. 318(4) (2000), 837-847.

[16] N. Nakayama, Zariski-decomposition and abundance, MSJ Memoirs, 14. Mathematical Society of Japan, Tokyo, 2004.

[17] S. Payne, Stable base loci, movable curves, and small modifications, for toric varieties, Math. Z. 253(2) (2006), 421-431.

[18] Yu.G. Prokhorov and V.V. Shokurov, Towards the second main theorem on complements. J. Algebra. Geom. 18(1) (2009), 151-199. 
[19] V.V. Shokurov and S. Choi, Geography of log models: theory and application, Cent. Eur. J. Math. 9(3) (2011), 489-534.

[20] S. Takayama, On the uniruledness of stable base loci. J. Differ. Geom. 78(3) (2008), 521-541.

Department of Mathematics, University of California, Riverside, 900 University Ave, RIVERSIDE, CA 92521, USA

Current address: Department of Mathematics, POSteCH, San 31, Hyojadong, Namgu, Pohang, Gyungbuk 790-784, Republic of Korea

E-mail address: sungrakc@postech.ac.kr 